\section{Versatile holographic data storage system for angular multiplexing with no upper limit of the angular sweep of the reference beam}

\author{
Mei-Li Hsieh \\ National Taiwan Normal University \\ Institute of Electro-Optical Science and Technology \\ Taiwan
}

\begin{abstract}
We propose and demonstrate a versatile holographic storage system using a single-mode polarizationmaintained (PM) fiber guided reference beam. By properly allocating the output coupler of the single-mode fiber, the system provides the ability of angular multiplexing recording with no upper limit of the angle tuning range of the reference beam. In addition, this system can easily achieve different holographic storage architectures including transmission, reflection, and 90-deg geometries. () 2005 Society of Photo-Optical Instrumentation Engineers.

[DOI: 10.1117/1.2047573]
\end{abstract}

Subject terms: holographic storage; angular multiplexing; volume hologram.

Paper 050350 LR received May 5, 2005; revised manuscript received Jul. 7, 2005; accepted for publication Jul. 15, 2005; appeared online Jul. 15, 2005; published online Sep. 15, 2005.

The main advantages of holographic storage technique are the very high volumetric storage capacity $\left(\sim 10^{12} \mathrm{bits} / \mathrm{cm}^{3}\right)$ and fast readout speed $\left(\sim 10^{11} \mathrm{bits} / \mathrm{sec}\right)$ (Refs. 1-4). The high storage capacity of volume holograms is attributed to the ability of recording multiple data pages at a single location through multiplexing techniques. ${ }^{5-7}$ Among them, angular multiplexing is the most commonly used scheme, and the reference beams for each page should be incident at the same position on the recording medium with different incident angles. In conventional structures, the direction of the reference beams can be controlled by use of a rotation stage and a telescope imaging system. ${ }^{1,3}$ The most serious problem of this optical system is that the total angle of multiplexing of the reference beam is limited by the aperture size in the telescope system. In terms of the geometric optics factors, the total number of data pages that can be recorded at one material location is equal to the total angle of multiplexing divided by the angular separation between two neighboring pages. Since the minimum angular separation is determined by the Bragg condition, the maximum storage capacity of each material spot is limited by the total angle of multiplexing, which is limited by the aperture size of the reference beam. For example, the Bragg condition of the commercial 1-mm holographic plate with $M / \#=10$ (Inphase Tech.: HDS2000) is estimated to be $0.1 \mathrm{deg}$. Suppose the numerical aperture (NA) lens of the reference beam is 0.5. To obtain good

0091-3286/2005/\$22.00 @ 2005 SPIE reference beam quality, we use only the central region of the lens, which is assumed to be $20 \mathrm{deg}$. From the geometric optics point of view, the storage capacity for angle multiplexing is limited to 200 pages. From the material point of view, the recording capability is 1000 pages, if the diffraction efficiency for each page is $1 \times 10^{-4}$. This number far exceeds that of the geometrical optics limitation. Apparently, if we want to fully utilize the material capacity, we should increase the lens aperture of the reference beam. This will inevitably increase the volume of the telescope to become bulky and impractical. Therefore, it is important to find a new way for increasing angle multiplexing without increasing volume.

On the other hand, different storage materials are suitable only for particular recording geometries. We require different recording geometries for characterization of different materials. However, conventional systems consisting of discrete optical elements are not easy to change, and alignment of the reference beams for angular multiplexing is particularly a dedicated procedure. Thus, it will be very useful to have a unique way for converting holography geometries without moving the optics of the reference beam.

In this letter, we propose a novel holographic storage system that has no limit for the total range of angle multiplexing, and the reference beam is guided by a single-mode polarization-maintained (PM) fiber from the laser source toward the storage medium. The recording medium is fixed at the central spot of a rotation stage, and the output coupler of the fiber is fixed at the circular rim of the rotation stage, which can rotate with respect to the center of the stage. Angle multiplexing is achieved simply by rotating the output coupler of the fiber around the recording spot. In principle, the angle tuning range can be as large as $360 \mathrm{deg}$. Therefore, this novel method provides a compact system for angle multiplexing holography with no upper limit of angle tuning. An additional merit of this system is that, by adjusting the position of the output coupler of the fiber, the system can easily achieve the transmission, reflection, and 90-deg geometries for holographic storage. Thus, our system could also be a compact and versatile test stand for holographic material research.

Figure 1 shows a schematic diagram of our system. A laser beam $(\lambda=532 \mathrm{~nm})$ is split into the object and refer-

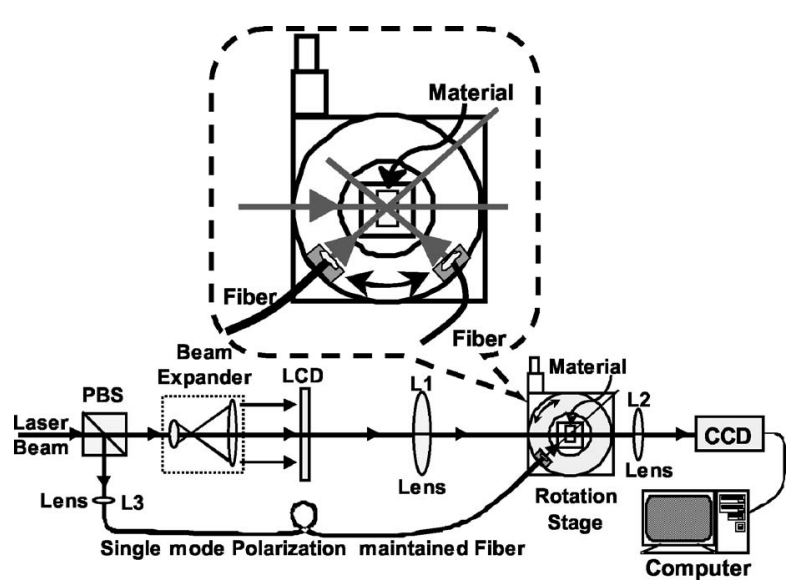

Fig. 1 Schematic diagram of the compact holographic storage system. 
ence beams by a polarizing beamsplitter (PBS). The object beam is expanded and collimated to illuminate a liquid crystal display as the input device. A two-dimensional CCD is used to detect the reconstructed image. The reference beam is coupled into a fiber (part number: Newport F-SPAOPT) by lens $L_{3}$, and guided to an output coupler, which is mounted on the rim of a precision rotation stage. The reference beam is collimated by a lens and aligned toward the center of the rotation stage. The recording medium is mounted and fixed at the central position of the stage, which is separated from the stage. Thus, the medium does not move and only the incident angle of the reference beam changes when the rotation stage is rotated. This is exactly the reference beam that is required for the angular multiplexing recording.

In fact, any rotation stage with a circular rotating ring can be used for this purpose as long as the holder of the recording medium is fixed at the central position and separated from the rotating ring. Here, we used Newport PM500-360 with the 0.0006-deg rotation accuracy. Since the rotation range is $360 \mathrm{deg}$, the transmission, reflection, and 90-deg geometries can be realized simply by rotating the fiber output coupler. Thus, this system provides the capability of angle multiplexing with no limit of angle sweeping for the reference beam.

In experiments, for the transmission geometry, the output fiber coupler was rotated to the same side of the recording material as the object beam (shown in Fig. 1). The recording angle between the reference and the object beams is $45 \mathrm{deg}$ in air. The recording material is a 5 -mm-thick $\mathrm{LiNbO}_{3}$ crystal. The diffraction efficiency is measured as a function of the deviation angle of the reading beam, and is shown by the circled spots in Fig. 2(a). From this curve the angular selectivity is about $0.01 \mathrm{deg}$. Since the reference beam comes from a single-mode fiber, the speckle effect is limited and thus the angular selectivity should be similar to that of the plane wave reference beam. ${ }^{7}$ The solid curve of Fig. 2(a) shows the theoretical curve of Bragg selectivity, ${ }^{8}$ and the two results match well.

When the output end of the fiber is rotated to the adjoining side of the object beam, 90-deg recording geometry can be achieved. In this case, the recording medium is a 45 deg-cut $\mathrm{LiNbO}_{3}$ crystal with $10 \times 10 \times 10 \mathrm{~mm}^{3}$ dimensions.

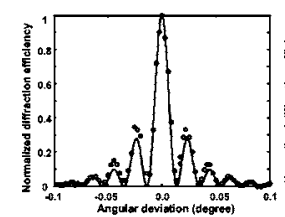

(a)

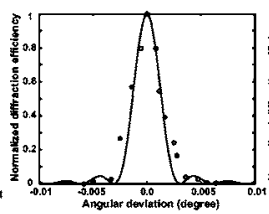

(b)

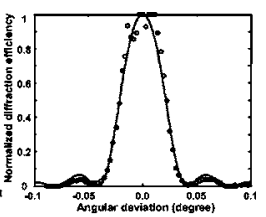

(c)
Fig. 2 Diffraction efficiency as a function of the angular deviation with different thickness of the recording material for the (a) transmission, (b) 90-deg, and (c) reflection hologram. The circled spots represent the measured data, and the solid curve represents the theoretical simulation.
The measured data are shown as dots in Fig. 2(b) and have no clear nulls of the sinclike function because the crystal has an absorption coefficient of $0.2 \mathrm{~mm}^{-1}$. The effect of the linear absorption induces a tapering of the index grating, which expands the width of mainlobe and reduces the peak values of sidelobes of diffracted sinclike signal. ${ }^{9}$ By considering absorption, the theoretical results are shown as the solid curve in Fig. 2(b). The angular selectivity is about $0.004 \mathrm{deg}$ for both experimental and theoretical cases.

When the position of the fiber is rotated to the opposite side of the object beam, the system performs the reflection hologram recording. The recording material is a $1-\mathrm{mm}-$ thick PQ/PMMA photopolymer, and the incident angle between the two recording beams is $135 \mathrm{deg}$. The measured and theoretical data are shown in Fig. 2(c), and both results show the Bragg selectivity is about $0.04 \mathrm{deg}$ in this case.

In conclusion, we proposed and demonstrated a versatile holographic storage system that provides the capability of angle multiplexing with no limit of total tuning angle for the reference beam. By properly rotating the rotation stage, the system can easily achieve transmission, reflection, and 90-deg geometries for recording holograms. The Bragg selectivity on different materials with three recording geometries were measured and were shown to be reasonably coincident with the theoretical analysis. The results show that our system could be a versatile platform for investigating holographic storage. Another advantage of our system by using a single-mode fiber is that the additional effects induced by the multimode fiber, such as speckle, stability, and ruggedness can be minimized. ${ }^{10}$

\section{Acknowledgments}

This research is supported by a grant from the National Science Council of ROC under Contract No. NSC 93-2215E-003-001. The author would like to thank Prof. Ken Hsu for the helpful discussion and support in this work.

\section{References}

1. G. T. Sincerbox, Ed., Selected Papers on Holographic Storage, SPIE Milestone Series, Vol. MS95, SPIE Press Bellingham, WA (1995).

2. D. Psaltis and F. Mok, "Holographic memories," Sci. Am. 273(5), 70-76 (1995).

3. IBM Holographic Optical Storage Team, "Holographic storage delivers high data density," Laser Focus World 36(12), 123-127 (2000).

4. L. Hesselink, S. S. Orlov, and M. C. Bashaw, "Holographic data storage systems," Proc. IEEE 92(8), 1231-1280 (2004).

5. D. Psaltis, M. Levene, A. Pu, G. Barbastathis, and K. Curtis, "Holographic storage using shift multiplexing," Opt. Lett. 20(7), 782-784 (1995).

6. O. Matoba and B. Javidi, "Encrypted optical storage with angular multiplexing," Appl. Opt. 38(35), 7288-7293 (1999).

7. Y. H. Kang, K. H. Kim, and B. Lee, "Angular and speckle multiplexing of photorefractive holograms by use of fiber speckle patterns," Appl. Opt. 37(29), 6969-6972 (1998).

8. P. Yeh, Introduction to Photorefractive Nonlinear Optics, Chap. 2, Wiley and Sons, New York (1993).

9. S. Campbell, S.-H. Lin, X. Yi, and P. Yeh, "Absorption effects in photorefractive volume-holographic memory systems. I. Beam depletion," J. Opt. Soc. Am. B 13(10), 2209-2217 (1996).

10. B. Lee, S. Han, Y. Jeong, and J. Paek, "Remote multiplexing of holograms with random patterns from multimode fiber bundles," Opt. Lett. 29(1), 116-118 (2004). 